\title{
Androgen-forming stem Leydig cells: Identification, function and therapeutic potential
}

\author{
Yunhui Zhang ${ }^{\mathrm{a}, \mathrm{b} *}$, Renshan $\mathrm{Ge}^{\mathrm{b}}$ and Matthew P. Hardy ${ }^{\mathrm{b}}$ \\ ${ }^{a}$ Department of Environmental Health, Fudan University, Shanghai, 200032, China \\ ${ }^{\mathrm{b}}$ Population Council and The Rockefeller University, New York, NY 10021, USA
}

\begin{abstract}
Leydig cells are the primary source of testosterone in the male, and differentiation of Leydig cells in the testes is one of the primary events in the development of the male body and fertility. Stem Leydig cells (SLCs) exist in the testis throughout postnatal life, but a lack of cell surface markers previously hindered attempts to obtain purified SLC fractions. Once isolated, the properties of SLCs provide interesting clues for the ontogeny of these cells within the embryo. Moreover, the clinical potential of SLCs might be used to reverse age-related declines in testosterone levels in aging men, and stimulate reproductive function in hypogonadal males. This review focuses on the source, identification and outlook for therapeutic applications of SLCs. Separate pools of SLCs may give rise to fetal and adult generations of Leydig cell, which may account for their observed functional differences. These differences should in turn be taken into account when assessing the consequences of environmental pollutants such as the phthalate ester, diethylhexylphthalate (DEHP).
\end{abstract}

Keywords: Stem Leydig cell, testosterone, Fetal Leydig cell, Adult Leydig cell, Phthalate

\section{Introduction}

Stem cells are of interest because they possess the ability to self-renew and differentiate into a variety of cell types. The recognition that somatic stem cells can be isolated and are able to renew tissues has led to research on the use these cells in clinical therapy [1]. For example, bone marrow cells were first used successfully four decades ago, and cord blood stem cells more recently, in the past 10-15 years, in stem cell therapy. The therapeutic applications of these cells have included treatment of blood disorders such as leukaemia, multiple myeloma, lymphoma, and disorders with defective genes such as severe combined immune deficiency [2, 3].

${ }^{*}$ Corresponding author: Yunhui Zhang, MD, PhD, Population Council, The Rockefeller University, 1230 York Avenue, New York, NY 10021, USA. E-mail: yzhang @ popcouncil.org.
Stem cells can be classified on a functional basis, according to their potential for differentiation, as toti-, pluri-, or multipotent (Fig. 1) [4]. During mammalian development, the fertilized oocyte generates a complex organism comprising more than 200 different cell types. Fertilization of the egg and sperm lead to the formation of a blastocyst, the inner cell mass of which is removed to create an embryonic stem cell line. The cells of the inner cell mass (ICM) are pluripotent and will differentiate under appropriate culture conditions into the three lineages of multipotential stem cells: ectoderm, endoderm and mesoderm, from which all mature cells will develop. Examples include nervous tissue, pancreas and heart, respectively. Developmental potential results from the capability of early embryonic cells to proliferate and to differentiate into all cell lineages including the germ line. More recently, it has been shown that embryonic germ (EG) cell lines, established from primitive reproductive cells of the fetus, are functionally equivalent to embryonic stem cells (ES). 


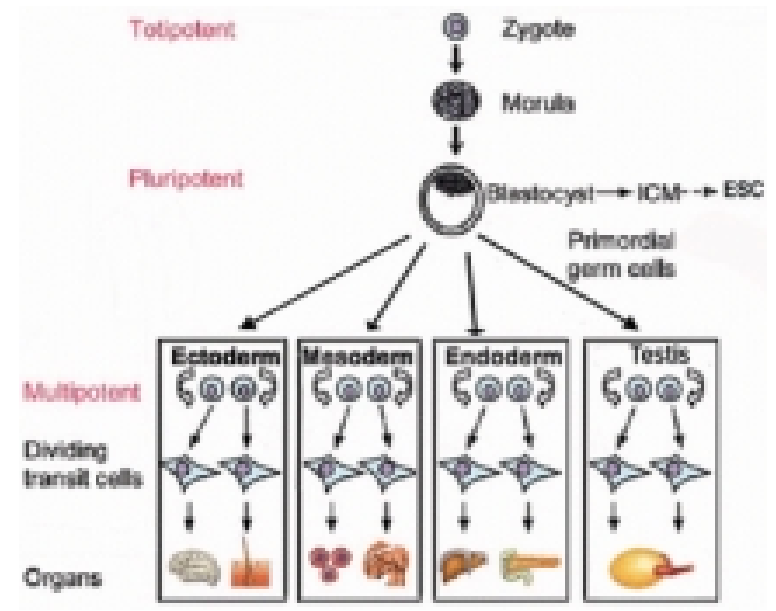

Fig. 1. The Stem Cell Hierarchy. At the top level is the totipotent original stem cell, the zygote, which results from syngamy of egg and sperm. Cell divisions of the zygote produce the morula stage of the embryo, the last point in embryogenesis where cells remain totipotent. Further divisions in the embryo and organization into a blastocyst is associated with the transition to multipotent stem cells in the inner cell mass (ICM), the primordial germ cells (PGCs, which in turn form the embryonic germ cells, EGs) and the three embryonic germ layers: ecto-, meso- and endoderm. The self-renewing aspect of multipotent stem cells in the three germ layers is indicated by the open arrows, and differentiation to daughter progeny that rapidly divide is shown by solid arrows. These daughter cells amplify the pool of cells available for differentiation and are classed as dividing transit cells. The final wave of terminal differentiation into cells that reside in tissues is shown. In the testis (right hand box), somatic and germ stem cell lineages both contribute to the formation of the testis (Modified from Czyz J, et al. Biol. Chem., 2003, with the permission of the publisher).

Totipotency, defined as the ability to generate an entire organism independently, is retained by the zygote and its early progeny up to the 8-cell stage of the morula. Pluripotency is the capability of a stem cell to create many, but not all, of the cell types in an organism. Cells in a developing embryo, totipotent at the beginning, lose this feature after several cell divisions as the organism develops and become pluripotent. Multipotent stem cells, finally, are those that can only give rise to cells of the tissue from which they were isolated. Therefore, based on the criteria of differentiation potential, embryonic stem cells (ESCs) are the least differentiated and, conversely, have the greatest potential and plasticity, as compared to multipotent stem cell lineages such as adult stem cells (ASCs) (bone marrow and tissue-specific stem cells), lineage-specific precursors, and terminally differentiated cells [5].

The distinguishing features of ESCs are their capacity to be maintained in an undifferentiated state indefinitely in culture and the potential to develop into ev- ery cell of the body. The ability of ESCs to develop into multiple cell types has drawn interest from investigators in regenerative medicine [6-8]. However, studies of ESCs have also highlighted problems associated with inefficient differentiation, tumorigenicity, immunogenicity and unresolved ethical issues surrounding the isolation of cells from human embryos. In this regard, ASCs provide an attractive alternative as they can be derived from the patient's own tissues, avoiding ethical issues and immunological rejection, and they are also non-tumorigenic [9]. ASCs could supply sufficient numbers of cells for current transplantation needs. Bone marrow stem cells and those ASCs (hematopoietic stem cells) derived from umbilical cord blood have been used, respectively, in regenerating heart muscle after myocardial infarcts and in the treatment of leukemia [10].

Adult stem cells probably reside in every tissue, having been detected in brain, bone marrow, peripheral blood, kidney, digestive tract epithelia, skin, retina, muscles, pancreas, and liver [11,12]. The ASCs have common features that enable them to produce identical daughter cells over the course of many rounds of cell division. This feature is often referred to as selfrenewal or clonogenicity. Another property of ASCs is their ability to give rise to precursors of descendents, and then terminally differentiated cells with specified morphological characteristics and functions [13]. In mature tissues, ASCs play a crucial role in maintaining local homeostasis by replacing dead or damaged cells as well as in the process of tissue remodeling [14].

More recently, it has also been suggested that testicular tissue can be regenerated from stem cell populations, including spermatogonial stem cells (SSCs) [15] and stem Leydig cells (SLCs) [16]. In the mammalian testis, continuous production of mature spermatozoa throughout life is maintained by a small population of SSCs. They are unipotent, and have the ability to form spermatozoa but no other cell lineages $[17,18]$. It is now possible to identify, isolate, maintain, expand the spermatogonia as cell lines, and to induce their differentiation in vitro [19]. When transplanted into the seminiferous tubules of an infertile male, SSCs can regenerate spermatogenesis in rats $[15,20,21]$.

Leydig cells are the primary source of testosterone in the male, and differentiation of Leydig cells in the testes is one of the primary events in the development of the male body and fertility. Most authors agree that SLCs are included among peritubular and perivascular fibroblast-like (also called mesenchymal) cells in the interstitial space of the postnatal testis. Between day 
7 and day 11, the SLCs commit to lineage specific differentiation, accompanied by further proliferation, ultimately leading to the formation of mature numbers of adult Leydig cells [22]. The clinical potential of SLCs for therapeutic androgen replacement has yet to be realized. Administration of exogenous androgen is now routine, but SLCs could have a relative advantage in that the release of testosterone would be subject to the body's innate regulatory cues and would thus be more physiological [23]. Thus, SLCs might be used to reverse severe age-related declines in testosterone levels in men. A more pressing case for clinical use might even be made with hypogonadal males.

Up until recently, it has not been possible to explore the potential of SLCs for stem cell therapy in the male, because these cells were unavailable. A lack of cell surface markers hindered attempts to obtain purified SLC fractions [24]. Once isolated, the properties of SLCs provide interesting clues for the ontogeny of these cells within the embryo. This review focuses on the source, identification and outlook for therapeutic applications of SLCs. In particular, we discuss their potential for treatment of androgen deficiency resulting from exposures to environmental pollutants, examining the anti-androgenic phthalate ester, diethylhexylphthalate (DEHP).

\subsection{Development of the Leydig cell lineage}

In rodents, Leydig cells exist as two generations, fetal and adult. The fetal Leydig cells (FLCs) differentiate from stem cells during gestation. They produce androgen required for masculinization of the male embryo as well as relaxin-like factor (RLF; also called insulin-like factor 3), which regulates the trans-abdominal phase of testicular descent $[25,26]$. The testis differentiates from the gonadal ridge primordium on day 10 (E10), and stem cells of the FLCs commit and differentiate into mature FLCs by E12. These cells are fully competent steroidogenically. The FLCs attain their highest steroidogenic activity 1 or 2 days prior to birth on day 19 of gestation, and the testosterone secreted is critical for male secondary sexual differentiation (i.e., development of the penis and sex accessory glands) [27]. The FLCs are terminally differentiated and do not divide. They remain in the testicular interstitium after birth, but rapidly involute [28], and the contribution of FLCs to postnatal androgen secretion is thought to be negligible.

After birth, the Leydig cell lineage can be conceptually divided into three developmental stages based on morphological and biochemical criteria [27]. It has been suggested that, in rats, the putative Leydig stem cells are present in the interstitium of the testis at birth, and that by 11 days postpartum, at least some of their progeny express Leydig cell-specific genes (i.e. $3 \beta$ hydroxysteroid dehydrogenase, $3 \beta$-HSD) and thus become committed to the Leydig cell lineage. The committed cells subsequently undergo phased transitions through progenitor and immature stages and ultimately become terminally differentiated adult Leydig cells (ALCs). This developmental sequence of Leydig cells has been defined only in the rat, but a similar progression is postulated for other species, including humans.

\subsubsection{Progenitor Leydig cells (PLCs)}

In the ALC lineage, stem cells do not directly convert into the terminally-differentiated daughter cells. Rather, they must first pass through transitional phases starting with the formation of PLCs. The PLCs are intermediate, transit amplifying populations that serve to supply differentiated cells in the numbers needed. As transit amplifying cells, PLCs arise from SLCs and divide a finite number of times until they become progressively more differentiated. The PLCs are stem celllike in that they are capable of maintaining their numbers by self-renewing divisions. However they differ from stem cells, which have a low mitotic rate and generate small numbers of progenitors, because they proliferate rapidly. As the first recognizable stage in the Leydig cell lineage, PLCs form from SLCs during days 12-28 postpartum. In addition to be being highly proliferative [29], PLCs express several of the mRNA and proteins associated with steroidogenic function, including cytochrome $\mathrm{P} 450$ cholesterol sidechain cleavage enzyme (CYP-11B1), 3 $\beta$-HSD, cytochrome $\mathrm{P} 45017 \alpha$-hydroxylase/ $\mathrm{C}_{17-20}$ lyase (CYP17), and luteinizing hormone receptor (LHR). They have negligible amounts of $17 \beta$-hydroxysteroid dehydrogenase 3 (17 $\beta$-HSD3), but have high expression levels of steroid metabolizing enzymes such as $5 \alpha$-reductase 1 and $3 \alpha$-hydroxysteroid dehydrogenase ( $3 \alpha$-HSD), and produce androsterone as their main androgen end product [30].

\subsubsection{Immature (ILCs) and adult Leydig cells (ALCs)}

During days 12 through 28 postpartum, PLCs gradually enlarge, become round, and reduce their proliferative capacity. This second transition results in the formation of another intermediate, ILCs, most commonly seen in the testis during Days 28 to 56 postpartum. In rats, ILCs have more smooth endoplasmic reticu- 
lum (SER) compared to PLCs and, in addition, contain cytoplasmic lipid droplets that support a high level of steroidogenic capacity. With the emergent increase of $17 \beta$-HSD3 activity in the presence of continued $5 \alpha$ reductase 1 and $3 \alpha$-HSD expression, $5 \alpha$-androstane$17 \beta, 3 \alpha$-diol (DIOL) is the predominant androgen synthesized [30]. ILCs undergo a final division before the transition to ALCs, which occurs by Day 56. ALCs are large, with an abundance of smooth endoplasmic reticulum, few lipid droplets, high levels of steroidogenic enzyme activity, and testosterone is the predominant androgen secreted due to the sharp decline in $5 \alpha$ reductase 1 expression [30]. ALCs appear at puberty and produce the testosterone required for the onset of spermatogenesis and the maintenance of adult male reproductive functions. ALCs comprise the predominant population of Leydig cells in the sexually mature testis.

Presently, it is still unknown whether FLCs and ALCs originate from a common stem cell. They are sufficiently different functionally to raise the question of whether their lineage is shared. A recent study demonstrated that thrombospondin 2 is mainly expressed in FLCs, and $3 \beta$-hydroxysteroid dehydrogenase 6 (3 $\beta$-HSD6) and $17 \beta$-hydroxysteroid dehydrogenase 3 (17 $\beta$-HSD3) are expressed in ALCs, thereby providing markers for the two Leydig cell generations [31]. In addition, the inhibitory guanine nucleotide-binding regulatory protein, which induces a rapid decline in LH-mediated signal transduction, is present in FLCs and undetectable in ALCs [32] .

SLCs have been isolated and have been found to meet two criteria for "stemness": (i) they are capable of self-renewal for months in vitro, implying that they are able to remain in the cell cycle throughout adult life without entering $G_{0}$ (and subsequently following the differentiation pathway) and (ii) they produce differentiated daughter cells [27].

\subsection{Identification and isolation of stem Leydig cells}

Identification of specific surface markers in SLCs has posed a technical challenge and could elucidate their ontogeny. Theoretically, SLCs are present in the testis only in small numbers and must be maintained by renewal cell divisions. Three distinctive characteristics would be expected for SLCs: (i) the ability to proliferate and expand indefinitely in vitro (self renewal) without showing signs of differentiation; (ii) the ability to differentiate (i.e., to express enzymes such as $3 \beta$ HSD and ultimately synthesize testosterone); and (iii) when transplanted into host rat testes, the ability to col- onize the interstitium and subsequently differentiate in vivo. Putative SLCs that were isolated from the neonatal testis at 7 days postpartum expanded their numbers during prolonged culture in vitro, and were induced to express proteins associated with Leydig cell differentiation, including LHR, steroidogenic acute regulatory protein (StAR), CYP-11B1, 3 $\beta$-HSD, and CYP-17. Moreover these cells were able to colonize a host testis that had been depleted of ALCs.

Morphologically, the putative SLCs are similar in morphology to PLCs. The putative SLCs are most readily harvested from the testis on day 7 postpartum, when the PLCs are not yet present, based on the presence of lineage-specific markers. The onset of steroidogenic enzyme and LHR gene expression occurs later in the PLCs which, like the SLCs, are spindle-shaped interstitial cells, on day 11 postpartum [33]. Many of the candidate markers of SLCs are receptors for hormones including leukemia inhibitory factor (LIF), platelet-derived growth factor receptor- $\alpha$ (PDGFR $\alpha$ ), and LHR.

\subsubsection{LIF and SLC self-renewal}

LIF is a member of the interleukin 6 family of cytokines and has been found to mediate self-renewal of stem cells [34]. LIF is required for long-term selfrenewal of neural stem cells and for maintenance of primordial germ cells in vitro. In the rat testis, the high concentration of LIF detected in testicular lymph supports the concept that peritubular cells are the principal source of testicular LIF [33]. LIF is detectable from 13.5 days of gestation onward and is predominantly expressed by peritubular myoid cells surrounding the seminiferous tubules. In the first week postnatally, the peritubular cells have a mesenchymal ultrastructure, appearing spindle-shaped by light microscopy. These cells form a two- to three-cell-layer-thick boundary tissue (lamina propria) adjacent to the basal lamina of the seminiferous epithelium. The putative SLCs are situated in the outermost layer of the boundary tissue, in the interstitial space, and therefore are likely to be targets of LIF. SLCs have been found to express LIFR, and LIF stimulates their proliferation in vitro. LIFR and its intracellular signaling partner subunit gp130 are expressed at their highest levels in PLCs relative to expression levels in ILCs and ALCs, thus linking the identified putative SLCs to later stages of LC differentiation [34]. 


\subsubsection{PDGFR $\alpha$ and commitment of SLCs to differentiation}

In mice, PDGFR $\alpha$ can be used as a Leydig cell lineage marker. PDGFR $\alpha$ is expressed at low levels in the mesenchyme of the mesonephros on day 11.5 of gestation in both sexes. It is also highly expressed in the coelomic epithelium and at the gonad-mesonephros border on day 11.5 again in both sexes. By day 12.5, expression is confined to the male and is very strong in the interstitial cells. After birth, PDGFR $\alpha$ has a more discrete distribution in the neonatal testis, and is located in the interstitial areas near peritubular cells. Finally, in adult testes, PDGFR $\alpha$ is localized exclusively in ALCs $[27,35]$. This change in the pattern of localization might suggest that $\operatorname{PDGFR} \alpha$-positive peritubular cells are in the stem cell niche of the Leydig cell lineage. Consistent with this idea, expression of PDGFR $\alpha$ was observed in immunopurified LHR-negative-PDGFR $\alpha$ positive cells obtained from 7-day-old rats. This indicates that PDGF signaling is necessary for stem cell commitment and differentiation in the Leydig cell lineage of mice and neonatal rats.

\subsubsection{Modulation of LH action on the differentiation of SLCs}

Testicular steroid hormone biosynthesis is a hormonally regulated, multistep process in which LH is known to play a crucial role as a trophic regulator. It has been established that development of steroidogenic capacity in Leydig cells requires stimulation by LH. In genetically hypogonadal LH-deficient GnRH hpg mice, for example, ALC numbers reach only $10 \%$ of control [36]. Similarly, although $3 \beta$ HSD-expressing PLCs are formed in LHR knockout mice, LCs fail to develop further and remain severely hypoplastic. These results indicate that factors other than LH may act on the putative SLCs to induce LHR expression. Evidence from mouse knockout studies suggests a role of for IGF1 and PDGF in SLC proliferation and differentiation. Within the SLC, the transcription factors GATA4 and steroidogenic factor 1 are involved in the induction of Leydig cell-specific gene expression.

\subsection{Gene expression changes during Leydig cell differentiation}

Changes in gene expression during development of Leydig cells have been investigated by cDNA array analysis using purified fractions of PLCs, ILCs, and ALCs. In the array, 1176 genes were present, representing 24 functional groups, including apoptosis and cell cycle proteins, extracellular regulators, heat shock, intracellular signaling proteins, metabolizing enzymes, oncogenes, transporters, and transcription factors. Of the 1176 genes, 513 were identified in PLCs, 423 in ILCs, and 581 in ALCs [37]. When the signal intensities for genes associated with PLCs were compared relative to ALCs, mRNAs corresponding for 107 known genes were down regulated by at least twofold. Among them, RCL $(R c l)$, a c-myc target gene, was the most abundant in PLCs, and was down regulated by fourfold in ALCs. In contrast, mRNA levels corresponding to 202 genes were increased by at least twofold during the transition from PLC to ALC, which was consistent with the increase of Leydig cell steroidogenic capacity during conversion of PLCs into ALCs. For example, genes encoding testosterone biosynthetic enzymes were highly expressed in ALCs with CYP-17 ranked number 1, 3 $\beta$-HSD1 number 4 , and CYP-11A1 number 17 [37]. The developmental patterns of gene expression are grouped in Fig. 2.

\section{Reproductive toxicants and Leydig cell lineage}

Mammalian spermatogenesis takes places within the avascular seminiferous tubules of the testis. Specialized tight junctions, which form between adjacent Sertoli cells at the time of puberty, divide the tubular space into the basal and adluminal compartments, and create a "blood-testis" barrier that restricts passage of substances and ions from the circulation. However, many chemicals are known to cause testicular damage in the human male and experimental animals, such as cadmium chloride, phthalates, and biphenyl A. The targets of these testicular toxicants may include germ, Sertoli, peritubular, or Leydig cells. When Sertoli cells are exposed to toxicants such as 2, 5-hexanedione [22] or mono-(2-ethylhexyl) phthalate, the resulting damage is typically irreversible because these cells are not maintained by a stem cell pool. In contrast, Leydig cells have a comparatively greater ability to recover from exposures to toxicants, which might be attributable to the persistence of SLC populations in the testis throughout adult life [38].

A distinguishing characteristic of many reproductive toxicants is the ability to act differently depending on developmental stage. An agent might have more severe effects, for example, if it acts on a stem cell population rather than the mature cell type. Some agents, such as busulphan, kill stem cells (spermatogonia), whereas others, such as ethane dimethane sulphonate, 


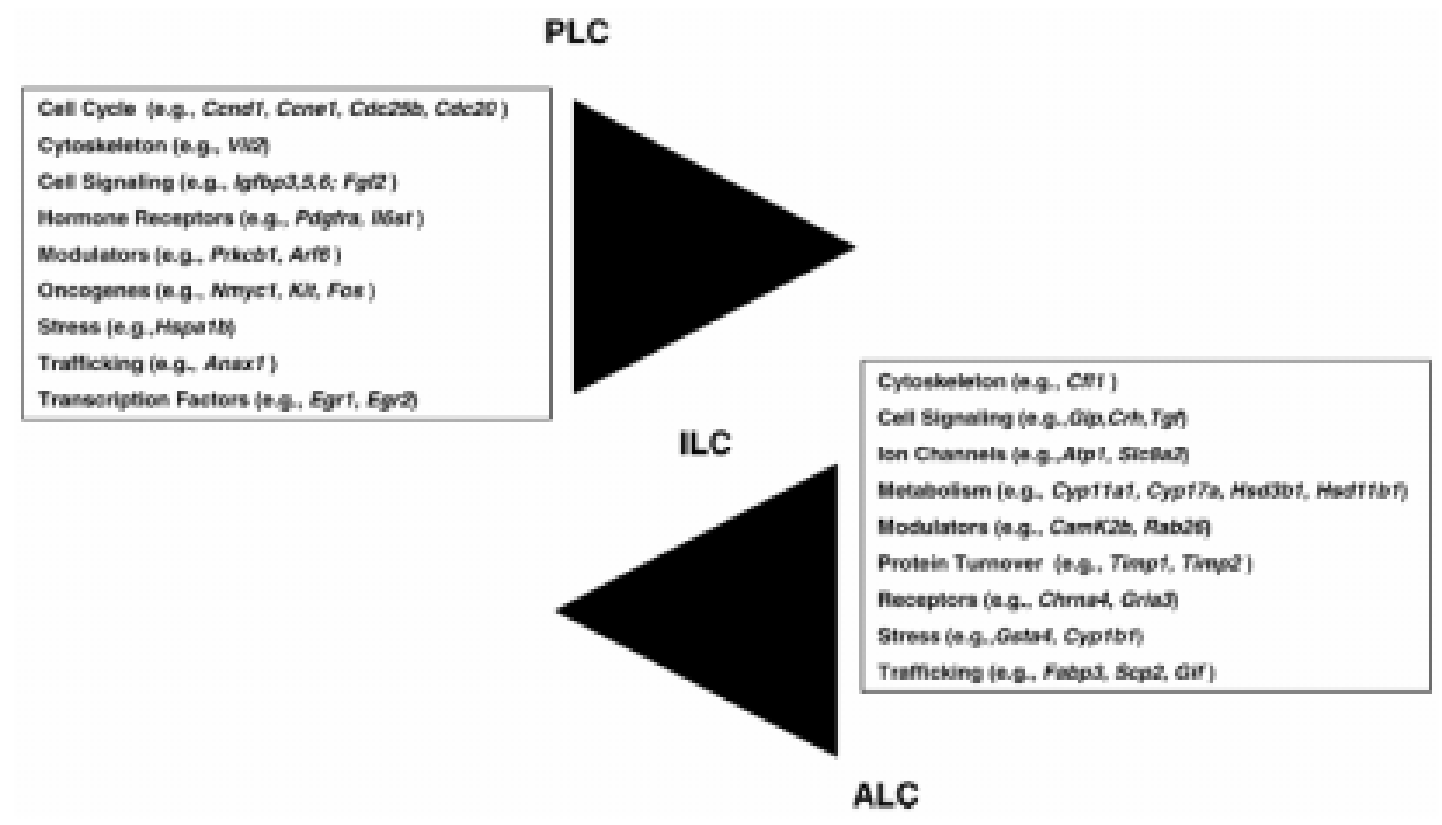

Fig. 2. Trends in gene expression during Leydig cell development. Among the more notable trends are decreased cell cycle (in the upper group), and increased steroid synthesis/metabolism (in the lower group), gene expression during the transitions from PLC to ILC and ILC to ALC. Potential markers of stemness were noted among the genes that are highly expressed in PLCs, which are the most immediate descendants of putative SLCs (From Ge RS, et al. Biol Reprod, 2005, with the permission of the publisher).

kill the mature terminally differentiated cell (i.e., Leydig cell), but leave the stem cell alone [38,39]. In some instances, however, sustained effects of a chemical are seen, which may indicate that toxic action is occurring on SLCs. For example, sustained declines in testosterone production after prenatal exposure to DEHP could indicate that stem cells of the FLCs are targeted [40].

Recent epidemiological evidence indicates that boys born to women exposed to phthalates during pregnancy have an increased incidence of genital malformations and spermatogenic dysfunction, signs of a condition referred to as testicular dysgenesis syndrome (TDS) [41]. TDS is thought to develop as a result of environmental factors that cause a testicular disturbance at an early fetal stage with a resultant spectrum of clinical testicular dysfunction, ranging from impaired spermatogenesis and genital malformations to increased risk for development of testicular cancer. Proposed environmental factors in the etiology of TDS include endocrine disrupting compounds such as the phthalates. Leydig cells have been classified as one of the main targets for phthalate toxicity in the body based on studies in rodents. The impact of phthalate exposures will be considered further to elucidate their differential toxicity during development.

\subsection{Phthalates and their endocrine disrupting effects}

Carlsen and co-workers reported that the quality of male sperm declined to $40 \%-50 \%$ in the past fifty years, drawing attention to potential biological effects related to environmental endocrine disruptors (EEDs). Exposures to EEDs are associated with reproductive tract anomalies including hypospadias (abnormal formation of the urethral meatus), and testicular maldescent [42]. According to the definition from the Endocrine Disruptor Screening and Testing Advisory Committee (EDSTAC), EEDs are exogenous substances that alone or as mixtures affect the function of the endocrine system causing adverse health effects. More than two hundred chemicals are considered to meet the criteria for classification as EEDs, including compounds such as pesticides, plasticizers, natural plant metabolites, detergents, and metals.

Phthalates are diester derivatives of phthalic acid used primarily as plasticizers to make plastic products more flexible. Certain plastics may contain up to $40 \%$ phthalate by weight. Phthalates do not become a permanent (chemically bonded) part of the plastic matrix during the manufacturing process, and they can and do leach from the plastic over time. From available exposure data, phthalates are found in many types of envi- 
ronmental specimens, including air, ground water, soil, sediment, seafood, etc. The concentrations of phthalates in those samples range from $10^{-9}$ to $10^{-1} \mathrm{mg} / \mathrm{L}$ $(\mathrm{mg} / \mathrm{kg})$. As a result, they have become ubiquitous in the environment, and people may be continuously exposed to low levels of phthalate.

Based in part on the significant environmental exposures, concerns have arisen over the possibility that phthalates exert reproductive and developmental effects in males and in the development of male offspring. The concerns have been particularly acute with respect to the most abundant phthalates, di-n-butyl phthalate (DBP) and di-2-ethylhexyl phthalate (DEHP). Animal data suggest a broad spectrum of health outcomes associated with phthalate exposure including developmental toxicity (cleft palate, decreased pup weight, testicular damage), endocrine disruption (testicular toxicity, decreased sperm motility, decreased fertility, decreased milk synthesis), and carcinogenicity $[43,44]$. Important windows of sensitivity to phthalate exposure occur in utero and during lactation [45]. There is no consensus on phthalate exposures and male reproductive toxicity in humans, most likely due to insufficient epidemiological data. In one report of Agency for Toxic Substances and Disease Registry (ATSDR), the investigators could not find a quantitative association between DBP concentration in semen and spermatozoa density. However, a cross-sectional study conducted in Shanghai shows that positive association exists between the incidence of sperm malformations and DEHP concentration in semen [46].

\subsection{Phthalate toxicity in Leydig cells}

The study of Leydig stem cell biology may also serve to enhance our understanding of reproductive toxicology. For example, phthalate exposures have been shown to induce cryptorchidism, hypospadias, impaired spermatogenesis, and reduced male fertility in rats [40]. Phthalates act not only in the seminiferous tubule, but are Leydig cell toxicants. Both FLC and ALC generations may be affected depending on the timing and duration of exposures, and the mechanisms of phthalatemediated toxicity may differ between the pre- and postnatal lineages. In this regard, the use of SLC cultures may allow the identification of the specific early risks of phthalate exposures, as well as the temporal nature and mechanism of the toxic effect.

Phthalates are now present virtually everywhere in the environment, and the prevalent human exposures are of particular concern. Recently, a reduction of the anogenital distance (AGD), an androgen dependent parameter of male sexual development, was observed in infant boys with increasing levels of MBP, MEP, monobenzyl and mono-isobutyl phthalate in maternal urine samples during late-pregnancy [47]. Boys with a short anogenital index also showed a high prevalence of cryptorchidism and small genital size $[47,48]$. Phthalate monoester contamination of human breast milk has an inhibitory effect on the postnatal surge of reproductive hormones in newborn boys [49]. These correlations between exposure levels and adverse reproductive health outcomes are apparently preceded by a decrease in fetal and adult Leydig cell function, inducing lower testicular levels of testosterone [50].

A biphasic effect on both fetal and adult Leydig cell populations appears to be a characteristic of phthalate exposures $[40,51,52]$. At lower doses, phthalates increase testosterone production either by increasing Leydig cell numbers [51] or by directly stimulating testosterone production $[40,52]$. The NOAEL and LOAEL for stimulation of testosterone can be achieved for DEHP at 1 and $10 \mathrm{mg} / \mathrm{kg} /$ day [40,51], respectively. This low-dose stimulation by DEHP is also seen after inhalation exposures to DEHP at doses comparable to oral administration of $1-5 \mathrm{mg} / \mathrm{kg} /$ day [53]. This increase in testosterone production is sufficient to advance the timing of puberty as judged by an earlier onset of preputial separation [40]. In contrast, phthalates inhibit testosterone production in both fetal and adult Leydig cells when rats are exposed to higher doses. Male reproductive tract abnormalities, as well as depressed testosterone levels, are observed at 6,12, and 18 months of age following in utero exposure to DBP, suggesting that the disturbance of FLCs is associated with a sustained disturbance of steroidogenesis in adult Leydig cells.

The underlying mechanisms of the biphasic effects of phthalate on Leydig cells are not well understood. One of the causes of increased testosterone production may be DEHP-mediated Leydig cell hyperplasia induced by increased estrogen action. Aromatase expression and activity are increased in Leydig cells after phthalate exposure. This, in turn, could lead to abnormal estrogen action at the testicular level, which is a known cause of Leydig cell hyperplasia and Leydig cell tumors in rodents. Steroidogenic capacity normalized to Leydig cell numbers decreases after 28-day exposures to low doses of DEHP, and the observed increases in serum testosterone levels may be explainable by increased cell numbers, which is consistent with the elevated serum LH levels seen after treatment [52]. 
Phthalate effects in the male at high doses may involve the activity of peroxisome proliferators. Peroxisome proliferators secreted by hepatic cells could stimulate the peroxisome proliferator-activated receptors (PPARs) in Leydig cells. PPARs which are members of a nuclear receptor superfamily then act as heterodimers with the retinoid $\mathrm{X}$ receptor (RXR) to regulate transcription, and once activated, induce transcription of genes containing peroxisome proliferator response elements. However, the reproductive toxicity of phthalates cannot be entirely explained by the action of a $\operatorname{PPAR} \alpha$-mediated pathway, because $\operatorname{PPAR} \alpha$-null mice remain sensitive to DEHP-mediated reproductive toxicity. Another signaling pathway in Leydig cells that could be disrupted by phthalate exposure is mediated by the aryl hydrocarbon receptor. Fetal testes of animals treated with DBP in vivo have an altered pattern of gene expression, including increased expression levels of aryl hydrocarbon receptor, CYP-1B1, and epoxide hydrolase, as assessed by cDNA microarray, which may indicate an oxidative stress response [37]. In general, newborns in neonatal intensive care units are considered to have large exposures to phthalates by coming into contact with the plastics in medical supplies, which are favored because they can be readily sterilized and disposed of after single use. As SLCs are most abundant in the fetus and neonate, newborns and children are the most vulnerable populations for phthalate exposures. Mechanisms of phthalate-mediated toxicity in SLCs will require additional analysis.

\section{Summary}

With the advent of their identification and isolation, SLCs provide a new tool for research into Leydig cell ontogeny and toxicology. In the future, SLCs may also have clinical utility as a means of increasing testosterone levels. The use of testosterone supplementation for elderly men has increased markedly over the last decade due to a recognized gradual decline in serum testosterone [54], which may lead to decreased bone mass, muscle strength, and libido. Research in SLCs is in its infancy, and many studies remain to be done before the promise for therapeutical use can be realized. The goal would be to use these cells in transplants to enhance testosterone levels in hypogonadal boys and in aging men. Transplantation of SLCs could afford a more effective and less-costly systemic therapy as compared to a lifetime need for steroid supplementation. The initial paradigm would call for harvesting the
SLCs of an androgen deficient male (such as a man who is aging or exposed to an environmental reproductive toxicant), amplifying these stem cells in vitro, inducing differentiation in vitro, and then autologously implanting the mature Leydig cells back into the same individual. Compared with the routine application of exogenous androgen, the obvious advantage of SLC therapy is that the release of testosterone would be subject to the body's innate regulatory cues and would thus be more physiological. In the event that SLCs are depleted as a result of pathology or exposures to environmental toxicants, it may be possible to perform allogeneic transplants of SLCs from another person. Presently, alginate-poly-L-lysine-encapsulated Leydig cell microspheres have been employed as a novel method for the delivery of testosterone in vivo, which might be adapted to the SLCs to futher expand research into their suitability for clinical use.

\section{Acknowledgments}

The authors thank Dr. Lei Dong for assistance with the illustrations, and Ms. Chantal Sottas for contributing to several of the studies that were included in this review. Supported in part by grants from the NIH (HD32588) and the Bixby Foundation.

\section{References}

[1] N. Shanthly, M.R. Aruva, K. Zhang, B. Mathew and M.L. Thakur, Stem cells: a regenerative pharmaceutical, $Q J \mathrm{Nucl}$ Med Mol Imaging 50 (2006), 205-216.

[2] F.P. Barry and J.M. Murphy, Mesenchymal stem cells: clinical applications and biological characterization, Int J Biochem Cell Biol 36 (2004), 568-584.

[3] B.E. Strauer and R. Kornowski, Stem cell therapy in perspective, Circulation 107 (2003), 929-934.

[4] J. Czyz, C. Wiese, A. Rolletschek, P. Blyszczuk, M. Cross and A.M. Wobus, Potential of embryonic and adult stem cells in vitro, Biol Chem 384 (2003), 1391-1409.

[5] M. Tarnowski and A.L. Sieron, Adult stem cells and their ability to differentiate, Med Sci Monit 12 (2003), 154-163.

[6] C. Allegrucci and L.E. Young, Differences between human embryonic stem cell lines, Hum Reprod Update 13 (2007), 103-120.

[7] S. Filip, J. Mokrý, D. English and J. Vojácek, Stem cell plasticity and issues of stem cell therapy, Folia Biol (Praha) 51 (2005), 180-187.

[8] B.E. Tuch, Stem cells - a clinical update, Aust Fam Physician 35 (2006), 719-721.

[9] C. Denning and H. Priddle, New frontiers in gene targeting and cloning: success, application and challenges in domestic animals and human embryonic stem cells, Reproduction $\mathbf{1 2 6}$ (2003), 1-11. 
[10] L.G. Melo, A.S. Pachori, D. Kong, M. Gnecchi, K. Wang, R.E. Pratt and V.J. Dzau, Gene and cell-based therapies for heart disease, FASEB J 18 (2004), 648-663.

[11] B. Bussolati and G. Camussi, Adult stem cells and renal repair, J Nephrol 19 (2006), 706-709.

[12] C.E. Gargett, Uterine stem cells: what is the evidence? Hum Reprod Update 13 (2007), 87-101.

[13] K. Hishikawa and T. Fujita, Stem cells and kidney disease, Hypertens Res 29 (2006), 745-749.

[14] S. Chunmeng and C. Tianmin, Skin: a promising reservoir for adult stem cell populations, Med Hypotheses 62 (2004), 683-688.

[15] C. Brawley and E. Matunis, Regeneration of male germline stem cells by spermatogonial dedifferentiation in vivo, Science 304 (2004), 1331-1334.

[16] M.S. Davidoff, R. Middendorff, G. Enikolopov, D. Riethmacher, A.F. Holstein and D. Muller, Progenitor cells of the testosterone-producing Leydig cells revealed, Journal of Cell Biology 167 (2004), 935-944.

[17] H. Kubota, M.R. Avarbock and R.L. Brinster, Spermatogonial stem cells share some, but not all, phenotypic and functional characteristics with other stem cells, Proc Natl Acad Sci USA 100 (2003), 6487-6492.

[18] G. Sonpavde, T.E. Hutson and B.J. Roth, Management of recurrent testicular germ cell tumors, Oncologist 12 (2007), $51-61$.

[19] M.R. Wallenfang, R. Nayak and S. DiNardo, Dynamics of the male germline stem cell population during aging of Drosophila melanogaster, Aging Cell 5 (2006), 297-304.

[20] J. Ehmcke, K. Hubner, H.R. Scholer and S. Schlatt, Spermatogonia: origin, physiology and prospects for conservation and manipulation of the male germ line, Reprod Fertil Dev 18 (2006), 7-12.

[21] M. Kanatsu-Shinohara, N. Ogonuki, K. Inoue, A. Ogura, S. Toyokuni and T. Shinohara, Restoration of fertility in infertile mice by transplantation of cryopreserved male germline stem cells, Hum Reprod 18 (2003), 2660-2667.

[22] S.G. Haider, Cell biology of Leydig cells in the testis, in: International Review of Cytology, K. Jeon, ed., Academic Press, New York, 2004, pp. 181-241.

[23] M. Machluf, A. Orsola, S. Boorjian, R. Kershen and A. Atala, Microencapsulation of Leydig cells: a system for testosterone supplementation, Endocrinology 144 (2003), 4975-4979.

[24] L. Braydich-Stolle, N. Kostereva, M. Dym and M.C. Hofmann, Role of Src family kinases and N-Myc in spermatogonial stem cell proliferation, Dev Biol 304 (2007), 34-45.

[25] I.M. Adham, J.M. Emmen and W. Engel, The role of the testicular factor INSL3 in establishing the gonadal position, 36. Mol Cell Endocrinol 160 (2000), 11-16.

[26] S. Zimmermann, G. Steding, J.M. Emmen, A.O. Brinkmann, K. Nayernia, A.F. Holstein, W. Engel and I.M. Adham, Targeted disruption of the Insl3 gene causes bilateral cryptorchidism, Mol Endocrinol 13 (1999), 681-691.

[27] R.S. Ge, Q. Dong, C.M. Sottas, V. Papadopoulos, B.R. Zirkin and M.P. Hardy, In search of rat stem Leydig cells: Identification, isolation, and lineage-specific development, Proc Natl Acad Sci USA (2006).

[28] I. Huhtaniemi and L.J. Pelliniemi, Fetal Leydig cells: cellular origin, morphology, life span, and special functional features, 116. Proc Soc Exp Biol Med 201 (1992), 125-140.

[29] R.S. Ge and M.P. Hardy, Decreased cyclin A2 and increased cyclin G1 levels coincide with loss of proliferative capacity in rat Leydig cells during pubertal development, Endocrinology 138 (1997), 3719-3726.
[30] R.S. Ge and M.P. Hardy, Variation in the end products of androgen biosynthesis and metabolism during postnatal differentiation of rat Leydig cells, Endocrinology 139 (1998), 3787-3795.

[31] P.J. O'Shaughnessy, L. Willerton and P.J. Baker, Changes in Leydig cell gene expression during development in the mouse, Biol Reprod 66 (2002), 966-975.

[32] V. Eskola, A. Rannikko, I. Huhtaniemi and D.W. Warren, Ontogeny of the inhibitory guanine nucleotide-binding regulatory protein in the rat testis: mRNA expression and modulation of LH and FSH action, Molecular \& Cellular Endocrinology 102 (1994), 63-68.

[33] S.M. Mendis-Handagama and H.B. Ariyaratne, Differentiation of the adult Leydig cell population in the postnatal testis, Biology of Reproduction 65 (2001), 660-671.

[34] P.C. Heinrich, I. Behrmann, G. Muller-Newen, F. Schaper and L. Graeve, Interleukin-6-type cytokine signalling through the gp130/Jak/STAT pathway, Biochem J 334(Pt 2) (1998), 297314.

[35] R.V. Hoch and P. Soriano, Roles of PDGF in animal development, Development 130 (2003), 4769-4784.

[36] B.A. Cooke, Transduction of the luteinizing hormone signal within the Leydig cell, in: The Leydig Cell, A.H. Payne, M.P. Hardy and L.D. Russell, eds, Cache River Press, Vienna, IL, 1996, pp. 351-364.

[37] R.-S. Ge, Q. Dong, C.M. Sottas, H. Chen, B.R. Zirkin and M.P. Hardy, Gene Expression in Rat Leydig Cells During Development from the Progenitor to Adult Stage: A Cluster Analysis, Biol Reprod 72 (2005), 1405-1415.

[38] J.W. Laskey, G.R. Klinefelter, W.R. Kelce and L.L. Ewing, Effects of ethane dimethanesulfonate (EDS) on adult and immature rabbit Leydig cells: comparison with EDS-treated rat Leydig cells, Biol Reprod 50 (1994), 1151-1160.

[39] R. Sumathi, V. Sriraman, B.S. Kurkalli, F.F. Rommerts and A.J. Rao, Ethane dimethylsulphonate selectively destroys Leydig cells in the adult bonnet monkeys (Macaca radiata), Asian Journal of Andrology 1 (1999), 115-120.

[40] R.S. Ge, G.R. Chen, C. Tanrikut and M.P. Hardy, Phthalate ester toxicity in Leydig cells: developmental timing and dosage considerations, Reprod Toxicol 23 (2007), 366-373.

[41] N.E. Skakkebaek, E. Rajpert-De Meyts and K.M. Main, Testicular dysgenesis syndrome: an increasingly common developmental disorder with environmental aspects, Hum Reprod 16 (2001), 972-978.

[42] E. Carlsen, A. Giwercman, N. Keiding and N.E. Skakkebaek, Evidence for decreasing quality of semen during past 50 years, BMJ 305 (1992), 609-6013.

[43] R.M. David, Proposed mode of action for in utero effects of some phthalate esters on the developing male reproductive tract, Toxicol Pathol 34 (2006), 209-219.

[44] P.M. Foster, E. Mylchreest, K.W. Gaido and M. Sar, Effects of phthalate esters on the developing reproductive tract of male rats, Hum Reprod Update 7 (2001), 231-235.

[45] M. Ema, E. Miyawaki and K. Kawashima, Critical period for adverse effects on development of reproductive system in male offspring of rats given di-n-butyl phthalate during late pregnancy, Toxicol Lett 111 (2000), 271-278.

[46] Y.H. Zhang, L.X. Zheng and B.H. Chen, Phthalate exposure and human semen quality in Shanghai: a cross-sectional study, Biomed Environ Sci 19 (2006), 205-209.

[47] K. Marsee, T.J. Woodruff, D.A. Axelrad, A.M. Calafat and S.H. Swan, Estimated daily phthalate exposures in a population of mothers of male infants exhibiting reduced anogenital distance, Environ Health Perspect 114 (2006), 805-809. 
[48] S.H. Swan, K.M. Main, F. Liu, S.L. Stewart, R.L. Kruse, A.M. Calafat, C.S. Mao, J.B. Redmon, C.L. Ternand, S. Sullivan and J.L. Teague, Decrease in anogenital distance among male infants with prenatal phthalate exposure, Environ Health Perspect 113 (2005), 1056-1061.

[49] K.M. Main, G.K. Mortensen, M.M. Kaleva, K.A. Boisen, I.N. Damgaard, M. Chellakooty, I.M. Schmidt, A.M. Suomi, H.E. Virtanen, D.V. Petersen, A.M. Andersson, J. Toppari and N.E. Skakkebaek, Human breast milk contamination with phthalates and alterations of endogenous reproductive hormones in infants three months of age, Environ Health Perspect 114 (2006), 270-276.

[50] R.M. Sharpe, Hormones and testis development and the possible adverse effects of environmental chemicals, Toxicol Lett 120 (2001), 221-232.
51] B.T. Akingbemi, R. Ge, G.R. Klinefelter, B.R. Zirkin and M.P. Hardy, Phthalate-induced Leydig cell hyperplasia is associated with multiple endocrine disturbances, Proc Natl Acad Sci USA 101 (2004), 775-780

[52] R.S. Ge, G.R. Chen, Q. Dong, B. Akingbemi, C.M. Sottas, M. Santos, S.C. Sealfon, D.J. Bernard and M.P. Hardy, Biphasic effects of postnatal exposure to diethylhexylphthalate on the timing of puberty in male rats, J Androl 28 (2007), 513-520.

[53] M. Ma, T. Kondo, S. Ban, T. Umemura, N. Kurahashi, M. Takeda and R. Kishi, Exposure of prepubertal female rats to inhaled di(2-ethylhexyl)phthalate affects the onset of puberty and postpubertal reproductive functions, Toxicol Sci $\mathbf{9 3}$ (2006), 164-171.

[54] F.M. Kohn, Testosterone and body functions, Aging Male 9 (2006), 183-188. 


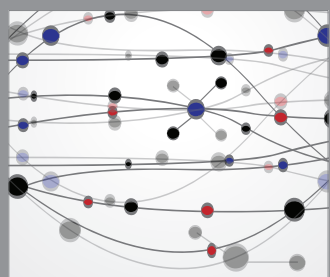

The Scientific World Journal
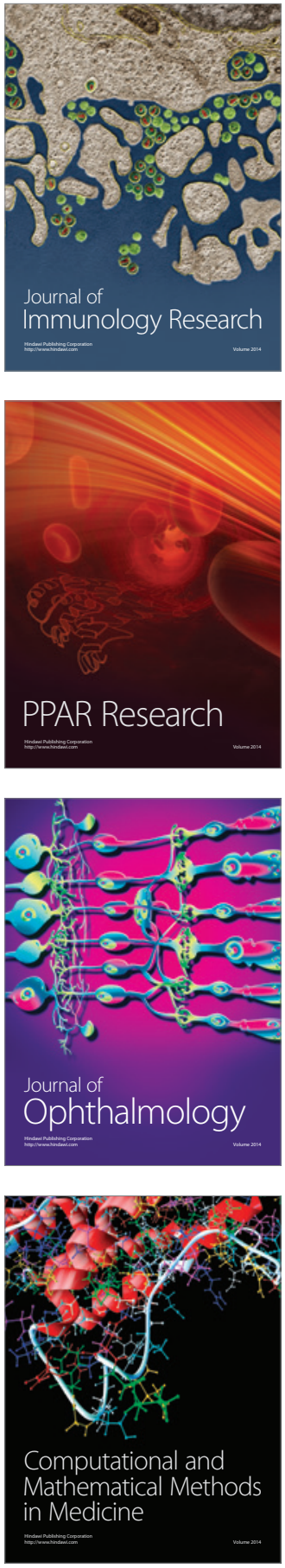

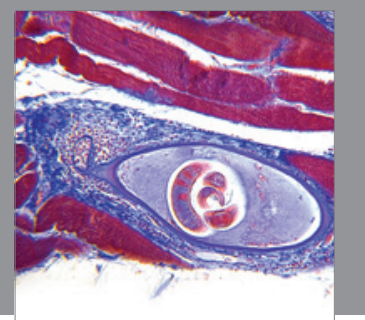

Gastroenterology

Research and Practice
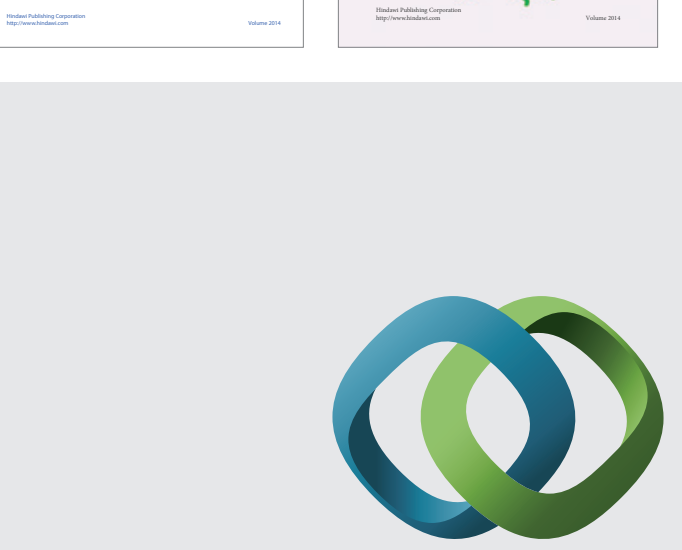

\section{Hindawi}

Submit your manuscripts at

http://www.hindawi.com
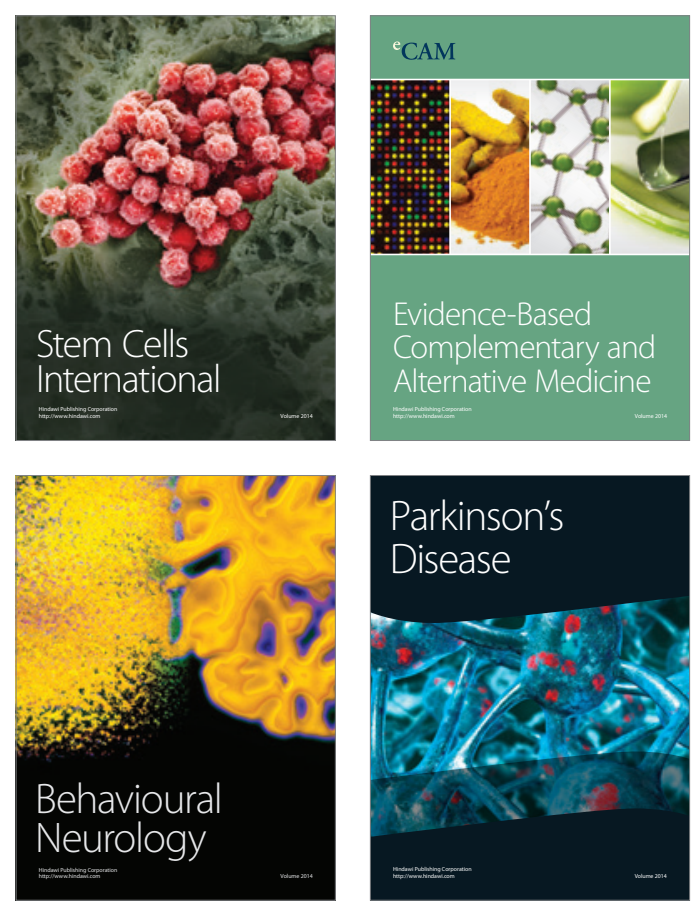

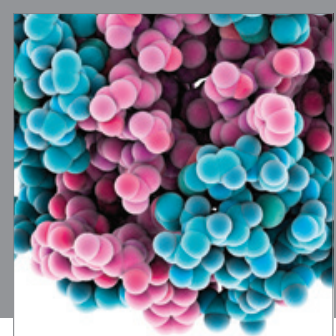

Journal of
Diabetes Research

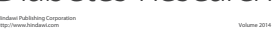

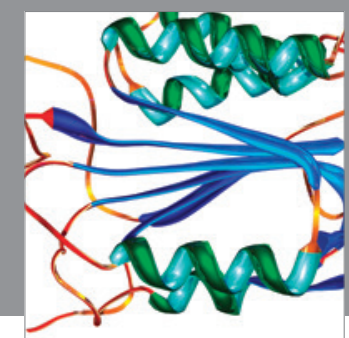

Disease Markers
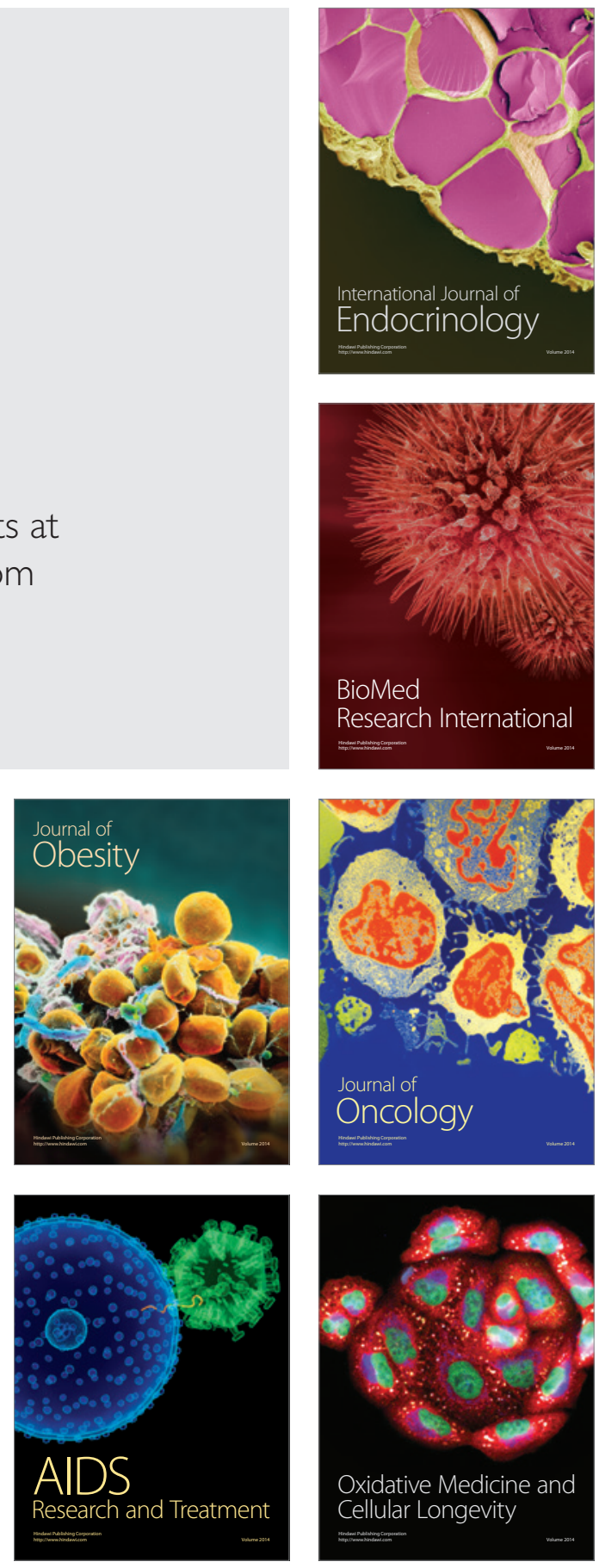